\title{
Influence of Individual Saliva Secretion on Fluoride Bioavailability
}

\author{
E.A. Naumova, P. Gaengler, S. Zimmer and W.H. Arnold* \\ Department für Zahn-, Mund- und Kieferheilkunde, Universität Witten/Herdecke, Alfred Herrhausen Strasse 50, 58448 \\ Witten, Germany
}

\begin{abstract}
The aim of this preliminary investigation was to compare the individual saliva secretion rate with the fluoride bioavailability in saliva after using sodium fluoride and amine fluoride. Methods: To assess oral fluoride kinetics 10 highly trained volunteers brushed their teeth with one of the formulations and saliva was collected. The amount of saliva was measured, and the fluoride content was determined. Data underwent statistical analysis using the Mann-Whitney-U test and Pearson correlation. The ex vivo experiment I included individual saliva collection of the same volunteers. Then the oral hygiene products were solved in equal amounts of whole saliva (ex-vivo experiment II), and the fluoride content was measured. Finally, both products were dispersed in distilled water (ex-vivo experiment III) to calculate the dissociation of both products in water. Results: In vivo results of fluoride content after $3 \mathrm{~min}$. tooth brushing demonstrated a negative correlation with saliva secretion: for $\mathrm{NaF} r=-0.695(\mathrm{p}<0.01)$ and for amine fluoride $\mathrm{r}=-0.446(\mathrm{p}<0.01)$. The invitro experiment I resulted for $\mathrm{NaF}$ in $251.7 \pm 22.4 \mu \mathrm{g} / \mathrm{g}$ fluoride and for amine fluoride in $171.7 \pm 14.4 \mu \mathrm{g} / \mathrm{g}$. Conclusions: Fluoride bioavailability of saliva after exposure to $\mathrm{NaF}$ was higher compared to amine fluoride. The individual secretion rate changes the fluoride content and normal secretors keep the fluoride availability longer.
\end{abstract}

Keywords: Fluoride, bioavailability, saliva, individual secretion, secretion rate, dentifrice.

\section{INTRODUCTION}

Fluoride has been a useful agent in caries prevention for a long time. It belongs to environmental factors, which influence the biomineralization of the teeth and their structural properties. [1,2]. The benefits of using fluoride to prevent caries have been known for many years, but a complete understanding of this mechanism is still being researched. There are different forms of fluoride application: Fluoride toothpastes, gels and mouth rinses are the main forms of self applied fluoride therapy. However, there is currently a debate regarding the appropriate use of fluorides $[3,4]$.

Numerous clinical trials have investigated the anti-caries effect of topical fluoride intervention. It appears that most of the trials have focused on topical fluoride in one form or another and that a small number of such trials have investigated the relative effectiveness of the main topical fluoride modalities [5, 6].

The effectiveness of fluoride oral hygiene products can be evaluated with morphological methods. Wiegand et al. [7] reported that the reaction of enamel concerning surface microhardness in caries-like demineralized enamel depends upon the fluoride ion concentration and increases with increasing fluoride concentration of the applied sodium fluoride gel. Therefore, the effectiveness of fluoride oral hygiene products depends upon the fluoride ion concentration. Nevertheless, there is still the question; which fluoride ion concentration is optimal for caries prevention. A decade ago Ogaard [8] stated that concentrated topical fluoride agents

*Address correspondence to this author at the Department für Zahn-, Mundund Kieferheilkunde, Universität Witten/Herdecke, Alfred Herrhausen Strasse 50, 58448 Witten, Germany; Tel: +492302926658; Fax: +492302926661; E-mail: wolfgang.arnold@uni-wh.de (such as in toothpaste, fluoride mouth rinses, gels, or varnishes) may have a different mechanism of caries inhibition compared to low-concentration applications (such as fluoridated water).

Saliva as the most important calcium reservoir for remineralization of teeth influences the bioavailability of fluoride for inhibiting caries lesions [9-11]. Salivary fluoride kinetics depends upon different factors: flow rates, age, stimulation effects, properties of fluoride containing products, volume and application time of these products, vehicle of fluoride, individual characteristics of saliva [12-14]. The interaction between these factors affects the process of fluoride clearance from the oral cavity. Ten Cate [15] noticed that efforts should continue to be directed towards improving our understanding of fluoride. Research started with laboratory studies to reveal the mode of fluoride action, attention later shifted to intra-oral studies and in situ product testing, and while for some topics, such as the efficacy of fluoride toothpastes, evidence is convincing, additional research is indicated to resolve remaining questions. One of the open questions since the pioneering work of Dawes and Weatherell [9] concerns the fluoride kinetics of new oral hygiene formulations. Furthermore it is of clinical interest to know how much fluoride is dissolved within the oral cavity after application of fluoride containing hygiene products during tooth brushing, how much of fluoride remains after expectorating the dentifrice slurry, and what is the optimal fluoride profile for caries protection.

This pilot study has therefore been carried out to verify the fluoride ionic concentrations in saliva immediately after tooth brushing by subjects with different saliva secretion rates using different fluoride formulations and to study fluoride ion clearance in the oral cavity. 


\section{MATERIALS \& METHODS}

\section{Subjects}

Ten healthy test subjects, 6 normal saliva secretors and 4 fast secretors, participated in this crossover study ( 8 male and 2 female subjects, 24 - 65 years of age). They consented after verbal and written information on the aim and performance of the investigation and also received written instructions and a schedule. Participants were further asked to avoid fluoride - rich food products such as tea, fish and specified mineral water during the period but had no restriction concerning drinking water. All test subjects were residents in the area with $\approx 2 \mu \mathrm{mol} / \mathrm{l}$ fluoride in the drinking water and normally used fluoride containing dentifrices twice daily. The participants had good oral health. The study protocol was approved by the Ethical Committee of the University of Witten/Herdecke, Germany (permission 21/2008).

\section{Fluoride Products}

Oral hygiene tablets DENTTABS ${ }^{\circledR}$ (proDentum Dentaltechnik GmbH, Berlin, Germany) contain $1450 \mu \mathrm{g} / \mathrm{g}$ fluoride from $\mathrm{NaF}$ The other ingredients according to the International Nomenclature of Cosmetic Ingredients (INCI) are microcrystalline hydroxyethyl cellulose, hydrated silica, sodium hydrogen carbonate, sodium lauryl sulphate, ascorbic acid, magnesium stearate, aspartame and mint flavor.

The dentifrice ELMEX ${ }^{\circledR}$ (Gaba, Lörrach, Germany) contains $1400 \mu \mathrm{g} / \mathrm{g}$ fluoride from amine fluoride. According to INCI the other ingredients are water, hydrated silica, hydroxyethyl cellulose, sorbitol and saccharine, peppermint oil, menthol, anethole, spearmint oil, limonene and titanium dioxide adjusted to $\mathrm{pH} 4.6$.

\section{Study Design}

In-vivo experiment: All experiments were carried out in the morning between 8 a.m. and 10 a.m. For baseline determination of fluoride saliva was collected from all test persons for 5 minutes by spitting into a plastic tube. The amount of saliva collected was weighed and the secretion rate determined and expressed as $\mathrm{g} / \mathrm{min}$. As there is a high individual variability in salivary secretion rate $[16,17]$ the test persons were characterized as normal $(0.3-0.6 \mathrm{~g} / \mathrm{min}$. $)$ and fast secretors (> $0.6 \mathrm{~g} / \mathrm{min}$.) according to the individual secretion rate. After baseline sample collection (T0), the test persons brushed their teeth with the assigned fluoride formulation for 3 min. under supervision of a dentist. For amine fluoride $1 \mathrm{~g}$ dentifrice was used, for $\mathrm{NaF}$ one tablet. The tablet had to be chewed before tooth brushing. Saliva samples (T1) were taken immediately after tooth brushing. All subjects repeated the two study arms five times, thus 10 samples were obtained per test person. The five cycles per subject for both fluoride formulations underwent statistical analysis. The wash-out period in-between each cycle was min. 3 days. During the wash out period the subjects used their personal oral hygiene procedure but brushed their teeth with non fluoridated dentifrice. The data of each visit of every test person were pooled and resulted in $\mathrm{n}=20$ for the fast secretors and $\mathrm{n}=30$ for normal secretors for each time interval measurement.

In-vitro experiment I: For determination of how much fluoride in $1 \mathrm{~g}$ dentifrice and 1 tablet was dissolved in saliva during 3 min. tooth brushing saliva samples from the same test person were taken 5 hours after tooth brushing by spitting into a plastic tube for $3 \mathrm{~min}$. According to earlier data the fluoride content of these samples was back to baseline [18]. The weight of the saliva samples of normal secretors and fast secretors was identical to the weight of sample T1 (immediately after brushing) from the same test person. $1 \mathrm{~g}$ dentifrice containing amine fluoride and 1 tablet containing $\mathrm{NaF}$ were dissolved in the saliva samples of every subject. All subjects repeated the two study arms for both fluoride formulations five times, and the data underwent statistical analysis.

In-vitro experiment II: The amount of fluoride dissolved in a standard amount of $5 \mathrm{~g}$ saliva was determined for both types of secretors. Saliva samples from the same test persons were taken 5 hours after tooth brushing. The weight of the saliva samples was in contrast to the in-vitro experiment I 5 g. $1 \mathrm{~g}$ dentifrice with amine fluoride and 1 tablet with $\mathrm{NaF}$ were dissolved in the saliva samples of each subject. All subjects repeated the two study arms for both fluoride formulations five times, and the data underwent statistical analysis. This experiment was performed in order to determine whether the fluoride bioavailability depends upon other factors of saliva than the salivary flow rate.

In-vitro experiment III: $1 \mathrm{~g}$ dentifrice with amine fluoride and 1 tablet with $\mathrm{NaF}$ were dispersed in 5 and then in 10 $\mathrm{ml}$ distilled water. The measurements for both fluoride formulations and for 2 concentrations (with 5 and $10 \mathrm{ml}$ water) respectively were 10 times repeated. This experiment was performed to compare the solubility of amine fluoride and $\mathrm{NaF}$ in saliva and water. The data underwent statistical analysis.

\section{Fluoride Determination}

After collection of whole saliva and weighting, the samples were centrifuged (B Centrifuge, Beckman Coulter $\mathrm{GmbH}$, Krefeld, Germany) for $10 \mathrm{~min}$ at.6000 rpm in microcentrifuge tubes. An aliquot of $1 \mathrm{ml}$ was taken and mixed with $1 \mathrm{ml}$ of a TISAB II buffer solution (Thermo Electron, Beverly, MA, USA). For fluoride ion distribution during the measurement a magnetic stick stirrer (size $2 \times 5 \mathrm{~mm}$ ) was used. The salivary fluoride content was analyzed using a fluoride-sensitive electrode (96- 09 Orion, Thermo Electron, Beverly, MA, USA).

For the measurement of the fluoride content the following analytical techniques were used: direct calibration and incremental techniques (the method of known addition for low ionic strength samples with a fluoride concentration less than $0.38 \mu \mathrm{g} / \mathrm{g}$ ).

Direct calibration was performed in a series of prepared standards of $0.4,4.0,40$ and $400 \mu \mathrm{g} / \mathrm{g}$ fluoride.

\section{STATISTICAL METHODS}

The obtained data were processed with the Statistical Package for Social Sciences (SPSS 15.0, Chicago, III., USA). As there was no normal distribution of the data the post-brushing values were compared with baseline levels using the non parametric Mann- Whitney- U test. Correlations were assessed with the Pearson correlation coefficient. 


\section{Fluoride content and secretion rate after using $\mathrm{NaF}$}

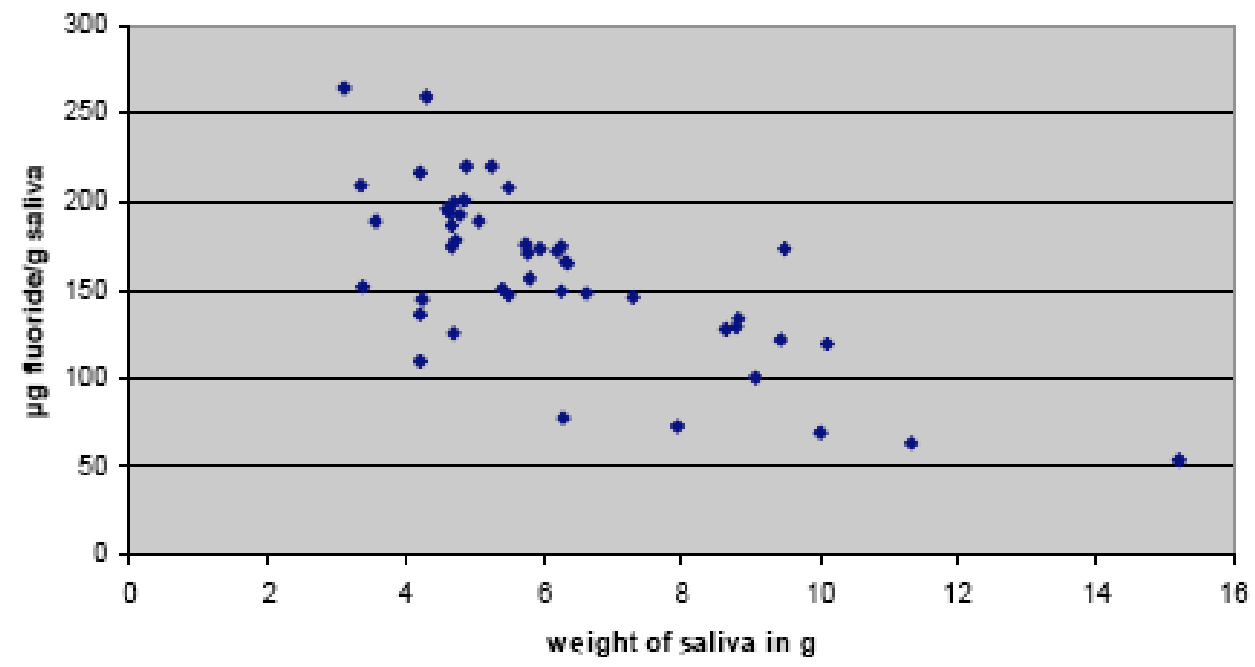

Fig. (1a). Fluoride content in saliva after 3 min. brushing of teeth wit NaF. There is a clear negative correlation between amount of saliva and fluoride content.

Fluoride content and secretion rate after using amine fluoride

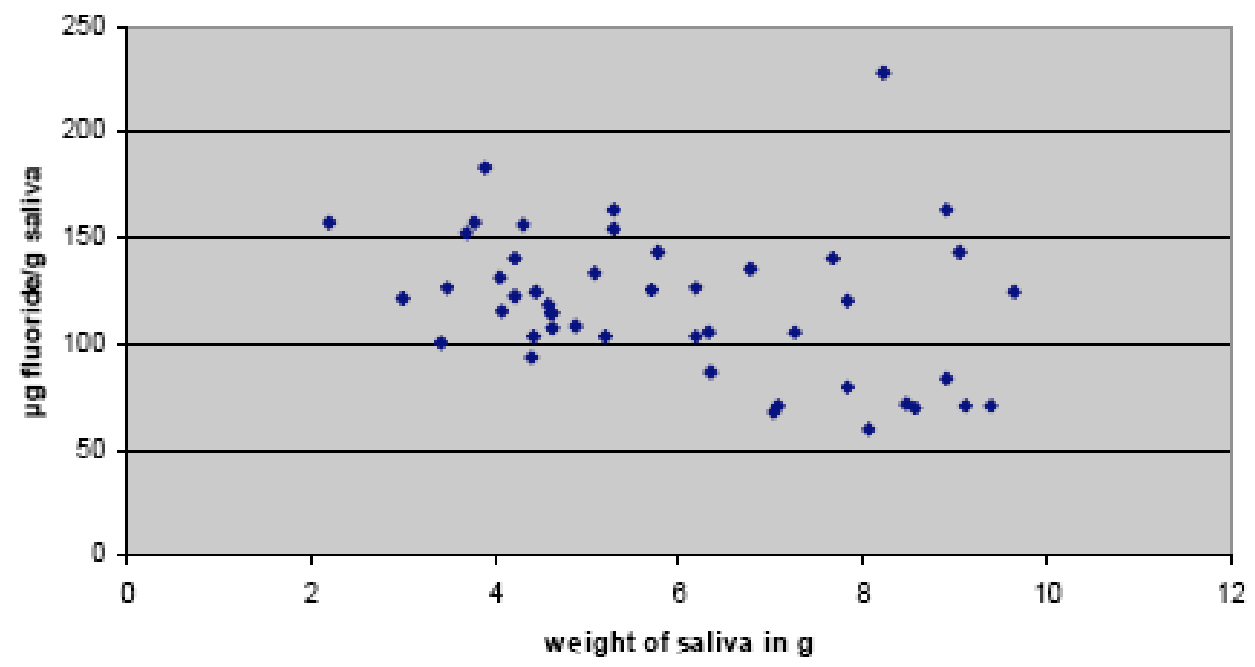

Fig. (1b). Fluoride content in saliva after 3 min. brushing of teeth with amine fluoride. There is also a negative correlation between the amount of saliva and fluoride content. However, the correlation is weaker.

The level of significance was determined at $\mathrm{p}<0.05$ and $\mathrm{p}<0.01$.

\section{RESULTS}

\section{Salivary Flow Rate}

The individual saliva secretion rate for the test persons was different, and the subjects were characterized as 6 normal secretors (mean secretion rate of unstimulated saliva $0.48 \pm 0.16 \mathrm{~g} / \mathrm{min}$.), and 4 fast secretors (mean secretion rate of unstimulated saliva $0.73 \pm 0.22 \mathrm{~g} / \mathrm{min}$ ).

The mean baseline value for fluoride concentration in whole saliva of all 10 subjects was $0.32 \pm 0.19 \mu \mathrm{g} / \mathrm{g}$ with a range from 0.002 to $1.06 \mu \mathrm{g} / \mathrm{g}$ (baseline).

\section{In-Vivo Experiment}

In these experiments the ratio of expectorated fluoride to the remaining fluoride content in the oral cavity was determined with regard to the salivary flow rate.

Fluoride content in the dentifrice/tablet slurry (saliva sample TI - expectorated immediately after tooth brushing) is presented in Fig. (1) for both, the fast secretors and the normal secretors, respectively. Immediately after tooth brushing a statistically significant increase was seen for both $\mathrm{NaF}$ and amine fluoride $(\mathrm{p}<0.05)$ but the increase was higher for $\mathrm{NaF}$. Fluoride concentration in saliva ranged from 52.5 to $266 \mu \mathrm{g} / \mathrm{g}$ (T1). Those with higher salivary flow rates tended to have saliva with a lower fluorde ion content. There was a 
Table 1. Saliva Fluoride Bioavailability in Relation to Saliva Secretion Rate Immediately after Tooth Brushing with NaF and Amine Fluoride (in - vivo Experiment)

\begin{tabular}{|c|c|c|c|c|}
\hline & \multicolumn{2}{|c|}{ NaF } & Amine Fluoride \\
\hline \hline $\begin{array}{c}\text { Saliva secretion rate } \\
(\mathrm{g} / \mathrm{min})\end{array}$ & $\begin{array}{c}\text { Mean } \pm \mathrm{SD} \\
(\mu \mathrm{g} / \mathrm{g} \mathrm{F})\end{array}$ & $\begin{array}{c}\text { Median; min/max } \\
(\mu \mathrm{g} / \mathrm{g} \mathrm{F})\end{array}$ & $\begin{array}{c}\text { Mean } \pm \text { SD } \\
(\mu \mathrm{g} / \mathrm{g} \mathrm{F})\end{array}$ & $\begin{array}{c}\text { Median; min/max } \\
(\mu \mathrm{g} / \mathrm{g} \mathrm{F})\end{array}$ \\
\hline $\begin{array}{c}\text { Normal secretion } \\
0.48 \pm 0.16\end{array}$ & $183.4 \pm 35.9$ & $182 ; 109 / 266$ & $130.9 \pm 29.6$ & $125 ; 77.6 / 233$ \\
\hline $\begin{array}{c}\text { Fast } \\
\text { secretion } \\
0.73 \pm 0.22\end{array}$ & $128.1 \pm 38.9$ & $131.5 ; 52.5 / 188$ & $103.7 \pm 32.4$ & $105 ; 59.4 / 164$ \\
\hline
\end{tabular}

The difference between the normal and fast secretors was significant at the $\mathrm{p}<0.05$ level.

Table 2. Saliva Fluoride Bioavailability in Normal and Fast Secretors for NaF and Amine Fluoride (in-vitro Experiment I)

\begin{tabular}{|c|c|c|c|c|}
\hline & \multicolumn{2}{|c|}{ NaF } & Amine Fluoride \\
\hline \hline $\begin{array}{c}\text { Saliva secretion rate } \\
(\mathrm{g} / \mathrm{min})\end{array}$ & $\begin{array}{c}\text { Mean } \pm \mathrm{SD} \\
(\mu \mathrm{g} / \mathrm{g} \text { F })\end{array}$ & $\begin{array}{c}\text { Median; min/max } \\
(\mu \mathrm{g} / \mathrm{g} \mathrm{F})\end{array}$ & $\begin{array}{c}\text { Mean } \pm \mathrm{SD} \\
(\mu \mathrm{g} / \mathrm{g} \text { F })\end{array}$ & $\begin{array}{c}\text { Median; min } / \mathrm{max} \\
(\mu \mathrm{g} / \mathrm{g} \text { F })\end{array}$ \\
\hline $\begin{array}{c}\text { Normal secretion } \\
0.48 \pm 0.16\end{array}$ & $251.7 \pm 22.4$ & $253.5 ; 200 / 296$ & $171.6 \pm 14.5$ & $174 ; 125 / 196$ \\
\hline $\begin{array}{c}\text { Fast } \\
\text { secretion } \\
0.73 \pm 0.22\end{array}$ & $203.7 \pm 18.7$ & $201 ; 175 / 243$ & $153.3 \pm 13.4$ & $151 ; 134 / 183$ \\
\hline
\end{tabular}

The difference between the normal and fast secretors was significant at the $\mathrm{p}<0.05$ level.

negative correlation of the salivary flow rate with the $\mathrm{F}^{-}$content at $\mathrm{T} 1$ for $\mathrm{NaF}(\mathrm{r}=-0.71 ; \mathrm{p}<0.01)$ and negative, but weaker correlation for amine fluoride $(r=-0.318 ; \mathrm{p}<0.029)$.

The mean expectoration rate of dentifrice/tablet slurry and saliva immediately after tooth brushing was $2.07 \mathrm{~g} / \mathrm{min}$ ranging from 1.04 to $5.07 \mathrm{~g} / \mathrm{min}$ in the study arm with $\mathrm{NaF}$ and $1.99 \mathrm{~g} / \mathrm{min}$ ranging from 0.73 to $4.12 \mathrm{~g} / \mathrm{min}$ in the study arm with amine fluoride.

Salivary F'bioavailability (T1) after 3 min. tooth brushing was significantly higher in normal secretors than in fast secretors for both products (Table 1).

\section{In-vitro Experiment I}

In in-vitro experiment $I$ it was determined how much fluoride in $1 \mathrm{~g}$ dentifrice and 1 tablet was dissolved in saliva during $3 \mathrm{~min}$. tooth brushing by test persons with different saliva secretion. Saliva fluoride concentration for amine fluoride and $\mathrm{NaF}$ in dependence from salivary secretion rate is presented in Table $\mathbf{2}$. The mean values showed that the fluoride concentration in whole saliva collected $5 \mathrm{~h}$ after tooth brushing with ex -vivo added fluoride formulations are statistically significantly higher and also statistically different for both amine fluoride and $\mathrm{NaF}$. The higher fluoride content in -vivo (Table 2) for $\mathrm{NaF}$ was reproduced ex -vivo in the individual amount of personal secretion rate, and normal secretors kept more fluoride in saliva.

\section{In-vitro Experiment II}

The fluoride content from the in-vitro experiment II (with the standard of $5 \mathrm{~g}$ individual saliva) for $\mathrm{NaF}$ was $237.1 \pm$
$30.7 \mu \mathrm{g} / \mathrm{g}$ and for amine fluoride was $165.7 \pm 16.4 \mu \mathrm{g} / \mathrm{g}$. No statistical differences were found for both types of saliva secretors.

\section{In-vitro Experiment III}

Mean fluoride concentration for amine fluoride in $5 \mathrm{ml}$ water was $184.8 \mu \mathrm{g} / \mathrm{g}$ and in $10 \mathrm{ml}$ water $110.7 \mu \mathrm{g} / \mathrm{g}$. Fluoride concentration for $\mathrm{NaF}$ in $5 \mathrm{ml}$ water was $254.6 \mu \mathrm{g} / \mathrm{g}$ and in $10 \mathrm{ml}$ water $137.5 \mu \mathrm{g} / \mathrm{g}$. Comparing these data with the results of in-vitro experiments I and II shows that the dissociation in water and saliva are very similar, but not identical. $\mathrm{NaF}$ exhibited again higher fluoride values $(\mathrm{p}<0.01)$.

\section{DISCUSSION}

The use of topically applied fluorides has increased over recent decades and fluoride containing toothpastes (dentifrices), mouth rinses, gels and varnishes are the modalities most widely used at present, either alone or in different combinations. By definition, the term 'topically applied fluoride' describes those delivery systems which provide fluoride to exposed surfaces of the dentition, at elevated concentrations, for a local protective effect and are therefore not intended for ingestion. Various modes of fluoride use have evolved, each with its own recommended concentration, frequency of use, and dosage schedule [5, 6].

Two decades ago Oliveby et al. [19] demonstrated that the fluoride excretion in human whole saliva is, unlike that of most others electrolytes, independent of flow rate. On the other hand it is also well known that the salivary fluoride clearance after topical application is highly site-specific and may have implications for the site-specifity of caries pro- 
gression [9]. Fluoride clearance is also dependent upon the fluoride concentration in the applied oral hygiene product [20].

Finally, Sjögren et al. [14] demonstrated a very slow fluoride clearance in dry mouth patients with considerably elevated fluoride concentrations in saliva. More recently it was shown that elevated fluoride products enhance remineralization of advanced enamel lesions [4] or may prevent the progression of incipient lesions [21].

Based on this knowledge the present pilot study was aimed at the evaluation of fluoride bioavailability in saliva in normal secretors and fast secretors following the use of different fluoride formulations: Sodium fluoride and amine fluoride as tablets, dissolved exclusively in saliva ( $\mathrm{NaF})$, and as dentifrice used as foam- saliva mixture (amine fluoride). Both secretor types were well discriminated over the whole experimental period. Although the basic salivary fluoride content varied considerably, the normal secretors exhibited a higher fluoride concentration in saliva / tablet slurry or saliva/ dentifrice foam than the fast secretors. The minimal flow rate was directly correlated to the highest fluoride concentration, and vice versa the maximal flow rate showed the lowest fluoride concentration.

Dissolving the two products in-vitro in the same individual amount of collected saliva demonstrated again higher fluoride contents for normal secretors, but the concentration was elevated compared to the in -vivo data. The same elevation has also been seen when the different fluoride formulations were dispersed in standardized $5 \mathrm{ml}$ saliva samples. In contrast to the complete dissolution of fluoride in individual amounts or standardised $5 \mathrm{ml}$ saliva samples outside the oral cavity two factors are contributing to relatively lower fluoride concentration immediately after tooth brushing: stimulation of saliva secretion due to the $\mathrm{pH}$ of products and to the mechanical influence, different distribution of slurry / foam within the oral cavity and retention in niches; whereas deglutition and expectoration was strictly avoided by the test subjects. Therefore, deglutition and expectoration had no influence on the fluoride concentration.

Finally, the dissolution of $\mathrm{NaF}$ and amine fluoride in distilled water reproduced different fluoride concentrations like in saliva. Obviously, the complex composition of saliva does influence the fluoride dissociation.

In all four experimental approaches, during tooth brushing, dissolving the fluoride compounds in individual amounts or in $5 \mathrm{ml}$ of collected saliva, and dissolving in distilled water, the flluoride concentration in the $\mathrm{NaF}$ arm of the study was significantly higher. Taking into account that the fluoride intake was nearly the same, means that the dissolution/dispersion of a dry product formulation in saliva during tooth brushing is contributing to an elevated bioavailability of fluoride.

Earlier results of a $\mathrm{pH}$ - cycling model demonstrated that the demineralization / remineralization balance, in particular remineralization, benefits from higher fluoride concentrations compared with traditional fluoride concentrations [22]. It could also bee shown, that a smaller volume at higher concentrations may increase the efficacy of fluoride [20]. This advantage of elevated fluoride products is well supported by microhardness measurements of the effect of different concentrations of $\mathrm{NaF}$ gels [7] and by the convincing results of transverse microradiography combined with the measurements of calcium uptake and loss [4].

The bioavailability of fluoride immediately after tooth brushing with the two oral hygiene products is different between individuals; however, there is a clear significantly higher fluoride concentration in normal secretors. Comparing both formulations, a novel oral hygiene tablet with a traditional dentifrice, it has been shown that the dissociation of sodium fluoride from a dry formulation exclusively in saliva results in higher concentrations and, therefore, in elevated bioavailability. This preliminary study demonstrated a high variability of the fluoride content in saliva after tooth brushing with different fluoride formulations. Individual caries prophylaxis has to take into account both factors: salivary secretion and the type fluoride application.

\section{AUTHORS CONTRIBUTIONS}

EAN: carried out the experiments

PG: responsible for the study design and correction of manuscript

SZ: supervisor of the project and study design

WHA: statistical calculations and manuscript writing

\section{ACKNOWLEDGMENTS}

The authors would like to thank Mrs. Susanne Haussman for her technical assistance in the fluoride measurements.

\section{REFERENCES}

[1] Robinson C. Self-oriented assembly of nano-apatite particles: a subunit mechanism for building biological mineral crystals. J Dent Res 2007; 86: p. 677-9.

[2] Vieira A, Hancock R, Dumitriu M, Schwartz M, Limeback H, Grynpas M. How does fluoride affect dentin microhardness and mineralization? J Dent Res 2005; 84: p. 951-7.

[3] Featherstone JD. Remineralization, the natural caries repair process:the need for new approaches. Adv Dent Res 2009; 21: p. 4-7.

[4] ten Cate JM, Buijs MJ, Miller CC, Exterkate RA. Elevated fluoride products enhance remineralization of advanced enamel lesions. $\mathrm{J}$ Dent Res 2008; 87: p. 943-7.

[5] Marinho VC, Higgins JP, Sheiham A, Logan S. Combinations of topical fluoride (toothpastes, mouthrinses, gels, varnishes) versus single topical fluoride for preventing dental caries in children and adolescents. Cochrane Database Syst Rev 2004: (1): CD002781.

[6] Marinho VC, Higgins JP, Sheiham A, Logan S. One topical fluoride (toothpastes, or mouthrinses, or gels, or varnishes) versus another for preventing dental caries in children and adolescents. Cochrane Database Syst Rev 2004: (1): CD002780.

[7] Wiegand A, Krieger C, Attin R, Hellwig E, Attin T. Fluoride uptake and resistance to further demineralization of demineralised enamel after application of differently concentrated acidulated sodium fluoride gels. Clin Oral Investig 2005; 9: 52-7.

[8] Ogaard B. The cariostatic mechanism of fluoride. Compend Contin Educ Dent 1999; 20: 10-7.

[9] Dawes C, Weatherell JA. Kinetics of fluoride in the oral fluids. J Dent Res 1990; 69 Spec No: 638-44; discussion 682-3.

[10] Featherstone JD. The science and practice of caries prevention. J Am Dent Assoc 2000; 131: 887-99.

[11] Margolis HC, Moreno EC, Physicochemical perspectives on the cariostatic mechanisms of systemic and topical fluorides. J Dent Res 1990; 69 Spec No: 606-13; discussion 634-6.

[12] Newbrun E. Topical fluorides in caries prevention and management: a North American perspective. J Dent Educ 2001; 65: 107883. 
[13] Schipper RG, Silletti E, Vingerhoeds MH. Saliva as research material: biochemical, physicochemical and practical aspects. Arch Oral Biol 2007; 52: 1114-35.

[14] Sjogren K, Birkhed D, Persson LG, Noren JG. Salivary fluoride clearance after a single intake of fluoride tablets and chewing gums in children, adults, and dry mouth patients. Scand J Dent Res 1993; 101: 274-8.

[15] Ten Cate JM. Fluorides in caries prevention and control: empiricism or science. Caries Res 2004; 38: 254-7.

[16] Blomgren J, Hasseus B. Inter- and intra-individual variations in saliva secretion rates:a comparison of unsupervised and supervised sample collection. Swed Dent J 2009; 33: 181-91.

[17] de Almeida Pdel V, Gregio AM, Machado MA, de Lima AA, Azevedo LR. Saliva composition and functions: a comprehensive review. J Contemp Dent Pract 2008; 9: 72-80.
[18] Naumova EA, Arnold WH, Gaengler P. Fluoride bioavailability in saliva using DENTTABS ${ }^{\circledR}$ compared to dentifrice. Centr Eur J Med 2010; 5: 375-380.

[19] Oliveby A, Ekstrand J, Lagerlof F. Effect of salivary flow rate on salivary fluoride clearance after use of a fluoride-containing chewing gum. Caries Res 1987; 21: 393-401.

[20] Duckworth RM, Stewart D. Effect of mouthwashes of variable $\mathrm{NaF}$ concentration but constant $\mathrm{NaF}$ content on oral fluoride retention. Caries Res 1994; 28: 43-7.

[21] Yamazaki H, Litman A, Margolis HC. Effect of fluoride on artificial caries lesion progression and repair in human enamel: regulation of mineral deposition and dissolution under in vivo-like conditions. Arch Oral Biol 2007; 52: 110-20.

[22] Gangler P, Kremniczky T, Arnold WH. In vitro effect of fluoride oral hygiene tablets on artificial caries lesion formation and remineralization in human enamel. BMC Oral Health 2009; 9: 25.

Received: May 26, 2010

(C) Naumova et al.; Licensee Bentham Open.

This is an open access article licensed under the terms of the Creative Commons Attribution Non-Commercial License (http://creativecommons.org/licenses/by-nc/3.0/) which permits unrestricted, non-commercial use, distribution and reproduction in any medium, provided the work is properly cited. 